\title{
MORPHOLOGICAL MODELING OF BAR DYNAMICS WITH DELFT3D: THE QUEST FOR OPTIMAL FREE PARAMETER SETTINGS USING AN AUTOMATIC CALIBRATION TECHNIQUE
}

\author{
Christophe Brière ${ }^{1}$, Alessio Giardino ${ }^{1}$ and Jebbe J. van der Werf ${ }^{1}$
}

\begin{abstract}
The modeling of bar dynamics is crucial for understanding coastal dynamics and shoreface nourishment evolution. Due to the complexity and variability of the physical processes involved, the formulations developed within the process-based numerical modelling system Delft3D for representing the forcing of the morphodynamic processes (waves, currents, sand transport) contain a high number of calibration parameters. Therefore, the setting up of any Delft3D computation requires a tedious calibration work, usually carried out manually and therefore by definition subjective. The aim of this work is the setting up of an automated and objective calibration procedure for Delft3D morphodynamic computations. A number of calibration parameters have been identified based on a careful sensitivity analysis. The calibration method named DUD (Does not Use Derivatives) is selected and coupled to a alongshore uniform Delft3D model. The validity of the implementation is shown based on synthetic tests (twin experiments). The validation test is carried out using field data collected at Egmond-aan-Zee (The Netherlands). This paper shows that the tool can be successfully used to calibrate Delft3D. However, further research is especially required to understand whether the computed parameters settings only simulate the best morphodynamic evolution of the bars or also describe properly the underlying physical processes.
\end{abstract}

Keywords: bar dynamics; Delft3D; parameter settings; calibration; coastal morphology; morphodynamic modelling

\section{INTRODUCTION}

\section{Background and objective}

The Dutch coast is yearly subject to a combination of beach and shoreface nourishments to account for local sand losses and to make sure the coast grows with sea level rise. Numerical modeling, associated to detailed field measurements, is commonly used to design shoreface nourishments and to assess their development and efficiency. However, the modeling of shoreface nourishments and of the breaker bars to which nourishments are attached is a complex task for modelers. This is due to a concurrency of different factors. First of all, the physical processes involved are very complex, especially in the cross-shore direction and their correct balance is crucial in order to determine the direction of transport, and the migration of nourishments and bars (Van Duin et al., 2004). In order to represent these complex processes, formulations have been developed including a high number of calibration coefficients, often empirical and site dependent (Ruessink et al., 2007). Due to these restrictions, predictions are reliable on a short time scale (weeks). Prediction accuracy decays going towards seasonal time scale, especially moving towards the inner bar (Van Rijn et al., 2003).

The aim of the work reported in the present paper is the setting up of an automated calibration tool to be used for morphodynamic simulations of bar dynamics and nourishments with Delft3D-MOR (Lesser et al., 2004). The site of Egmond aan Zee (The Netherlands) was chosen for setting up and testing the tool. The main requirement of the calibration procedure is to obtain reliable results in a reasonable computational time. This involves the choice of a suitable calibration technique and of a limited number of calibration parameters, which could lead to the best representation of the measured bathymetry.

The results of the study are a first step towards a general calibration guidelines for morphological simulations with Delft3D of breaker bar dynamics under different hydrodynamic and wave conditions, and for different locations. This could avoid, in the future, tedious calibration procedures for every new morphodynamic simulation.

\section{Methodology}

Several steps need to be carried out in order to set up and evaluate the calibration tool. At first, all free parameters in Delft3D-MOR are discussed, focusing on their relative effect on the bar morphodynamics (cf. section Modeling frame). The next step is the selection of a suitable calibration procedure (cf. section Calibration procedure). Different procedures are evaluated, leading to the choice of the DUD (Does not Use Derivatives) algorithm of Ralston and Jennrich (1978). Sensitivity

\footnotetext{
${ }^{1}$ Deltares, Department ZKS-MSD, Rotterdamseweg 185, 2629 HD Delft, The Netherlands
} 
tests with the different Delft3D free parameters are performed to reduce the choice of the relevant parameters to be used in the calibration tool. The calibration tool is then set-up and tested on a number of artificial experiments (twin experiments) with the aim of converging to a bathymetry developed under known parameter settings, varying the initial values of these parameters (cf. section Twin experiments). At last, the calibration procedure is applied to a field case at Egmond-aan-Zee for a 22-day period (cf. section Egmond-aan-Zee Field case).

More details on the set-up of the calibration procedure can be found in Giardino et al. (2010).

\section{MODELING FRAME}

\section{Delft3D model}

Simulations are carried out by means of the Delft3D model (research version, February 2009). In particular the following modules are used: the hydrodynamic module Delft3D-FLOW, the wave module Delft3D-WAVE, and the sediment transport module Delft3D-SED. Delft3D-FLOW and Delft3D-SED are now incorporated inside one module named Delft3D-MOR (Lesser et al. 2004).

The Delft3D-FLOW model solves the Navier Stokes equations for an incompressible fluid, under the shallow water and the Boussinesq assumptions. The time step for the hydrodynamic computation is selected equal to $12 \mathrm{~s}$. The superimposed effect of currents and waves on the bed shear stress is taken into account by means of the interaction model of Fredsøe (1984). Turbulence effects are computed by means of the K-epsilon model. Horizontal background eddy viscosity and diffusivity are set equal to $1 \mathrm{~m}^{2} / \mathrm{s}$. A value of $10^{-6}$ is used for the vertical background viscosity and diffusivity.

Wave heights are computed using the roller model (Reniers et al. 2004), included in the Delft3DFLOW module. The roller model consists of one balance equation for the short wave energy, and another one for the roller energy. Wave energy dissipation due to wave breaking is regarded as the only dissipative mechanism inside the balance equation for the short wave energy. Wave energy dissipation acts as a source term in the balance for the roller energy propagation. Dissipation due to wave breaking is computed according to the formulation of Roelvink (1993), which is an extension of the Battjes and Janssen model (1978). The breaker delay parameterization of Roelvink et al. (1995) is activated in the roller model. Input parameters for the roller model consist of mean wave direction and peak frequency inside the domain. Moreover, the wave energy at the boundary and a value equal to zero for the roller energy have to be prescribed at the open boundary. The mean wave direction used in the roller model of a 2DV model is obtained by applying Snell's law inside the domain while the peak period is assumed constant.

The sediment transport and morphodynamic computation are carried out by means of the Delft3D-SED module. The update expression of the TRANSPOR2004 formula (Van Rijn, 2007a, b) is used to calculate the bedload and suspended sediment transport. Bed shear stress calculation is based on the Van Rijn (2007a) roughness predictor. Sediment was assumed to be sandy with a D50 equal to $200 \mu \mathrm{m}$, and a sediment density equal to $2650 \mathrm{~kg} \cdot \mathrm{m}^{-3}$. The dry bed density was set equal to 1600 $\mathrm{kg} \cdot \mathrm{m}^{-3}$. Suspended sediment diameter at the beginning of the computation has a representative diameter equal to the D50. A minimum water depth equal to $0.2 \mathrm{~m}$ was assumed for sediment transport calculation. The transversal and longitudinal calibration factors for bed slope effects were respectively set equal to 1.5 and 1 .

\section{Free model parameters}

The fate of a model simulation is largely determined by the values of the free model parameters. These parameters reflect the uncertainty on how to model certain physical processes and provide the opportunity to tune models using measured data. This section summarizes the free model parameters in the Delft3D Roller, Flow and Transport module, which are considered the most uncertain and important given the scope of the study. More specifically for our applications five parameters have been selected: the slope of the wave front in the roller model, the tuning parameter for longitudinal slopes, the scaling factor for current and wave related suspended transport, and the calibration factor for ripples and megaripples in the roughness predictor.

The slope of the wave front, $\beta_{\mathrm{rol}}$, is an important parameter in the roller energy balance. It determines largely the rate of wave energy transferred to the roller and from the roller to the underlying water. Low values of $\beta_{\text {rol }}$ cause a delayed response. This parameter only has a small effect on the wave height predictions. However, the wave set-up can be influenced significantly. This parameter can be used to calibrate water levels and currents as it determines the cross-shore 
distribution of the surface shear stress due to wave breaking. Walstra et al. (2004) and Brière \& Walstra (2006) showed that the bar flattened considerably when using values larger than 0.1 . Decreasing values for $\beta_{\text {rol }}$ resulted in more pronounced inner and outer bars. Although in recent studies there was a lot of attention for breaker delay concepts, it seems that tuning of the roller model using $\beta_{\text {rol }}$ has a larger effect. It seems to provide a good handle to calibrate and control the behaviour of breaker bars.

Bedload is affected by bed level gradients. Two bed slope directions are distinguished: the slope in the initial direction of the transport (referred to as the longitudinal bed slope) and the slope in the direction perpendicular to that (referred to as the transverse bed slope). The longitudinal bed slope results in a change in the sediment transport rate and is computed using the method of Bagnold (1966). Walstra et al. (2004) showed that the longitudinal bed slope gradient had a very limited effect when applied within a realistic range (0 to 5). Larger values resulted in a significant unrealistic flattening of the profile.

The computed sediment transport contains four contributions: 1) current-related bedload, 2) wave-related bedload, 3) current-related suspended load and 4) wave-related suspended load. These contributions can be scaled using $f_{\text {bed,w }}, f_{\text {bed,w }}, f_{\text {sus }}$ and $f_{\text {sus, },}$, respectively. Except for the current-related suspended load, the contributions are directly multiplied with these factors. The sink and source terms of the advection-diffusion equation are multiplied by $f_{\text {sus }}$, and by this affecting the current-related suspended load. Brière \& Walstra (2006) found a strong effect of the scaling of the current-related suspended load $\left(f_{\text {sus }}\right)$ and the migration rate of the inner and outer bars, showing that this sediment transport process was dominant in the development of the beach profile. The coefficient changed the final shape, with a significant offshore migration with increasing $f_{\text {sus }}$. High values of $f_{\text {sus }}(0.6$ to 2.0) caused an unrealistic erosion of the upper part of the profile. However, the common practice of setting $f_{\text {sus }}$ to 1.0 seemed appropriate to reproduce properly the dynamics of the inner and outer bars. They also showed that the $f_{\text {bed }}$ coefficient did not influence the 1-year evolution of the profile, as currentrelated bedload is not important for bar dynamics compared to the current-related suspended load. They also investigated the effect of $f_{b e d, w}$ and $f_{\text {sus,w. }}$. Excluding the onshore bedload, resulted in an irregular profile and an enhanced offshore migration of the inner and outer bar. Including the transports, by applying factors in the range of 0.5 to 1.5 , led to a more realistic profile development. However, when the bed load factor was increased further, an enhanced flattening of the profile occurred, caused by bed slope effects on the transports. The influence of the bedload was limited when realistic scaling factors were used. Increasing $f_{\text {sus,w }}$ resulted in a reduction of the offshore bar migration. However, a significant smoothing of the profile is noticed for the highest $f_{\text {sus,w }}$ values, caused by the fact that the wave-related suspended transports are added to the bed load, which is affected by the bed slope gradients. Note: in the Delft3D version used in this study the bed slope only operates on the bedload and no longer on the wave-related suspended load.

The predictor of Van Rijn et al. (2004) (see also Van Rijn, 2007a) contains three contributions to the current-related roughness, associated with the presence of ripples, mega-ripples and dunes (only present in rivers). These contributions are scaled through the user-defined coefficients $f_{k s, r}, f_{k s m, r}$ and $f_{k s, d u}$, respectively. Brière \& Walstra (2006) tested the roughness predictor of Van Rijn. For low $f_{k s, r}$ and $f_{k s, m r}$ values, the outer and inner bars migrated further offshore compared to higher values. On the other hand, increasing $f_{k s, r}$ and $f_{k s, m r}$ led to a slight flattening and widening of the outer bar. The flattening of the inner bar was much more pronounced when increasing the $f_{k s, r}$ and $f_{k s, m r}$ values.

\section{Default values of the free model parameters}

Table 1 presents an overview of the (main) free parameters in the Roller, Flow and Transport module, together with their default value. Most of free model parameters have actually been treated as such in the sensitivity analysis performed by Walstra et al. (2004), and Brière \& Walstra (2006), except for the bottom friction factor, roller dissipation coefficient, horizontal background viscosity and the vertical background viscosity and diffusivity. Still, there is no general agreement on values for the roller slope, wave height-to-depth ratio, the horizontal background viscosity, scaling of the waverelated suspended load and the coefficient for the transverse bed slope effects. 


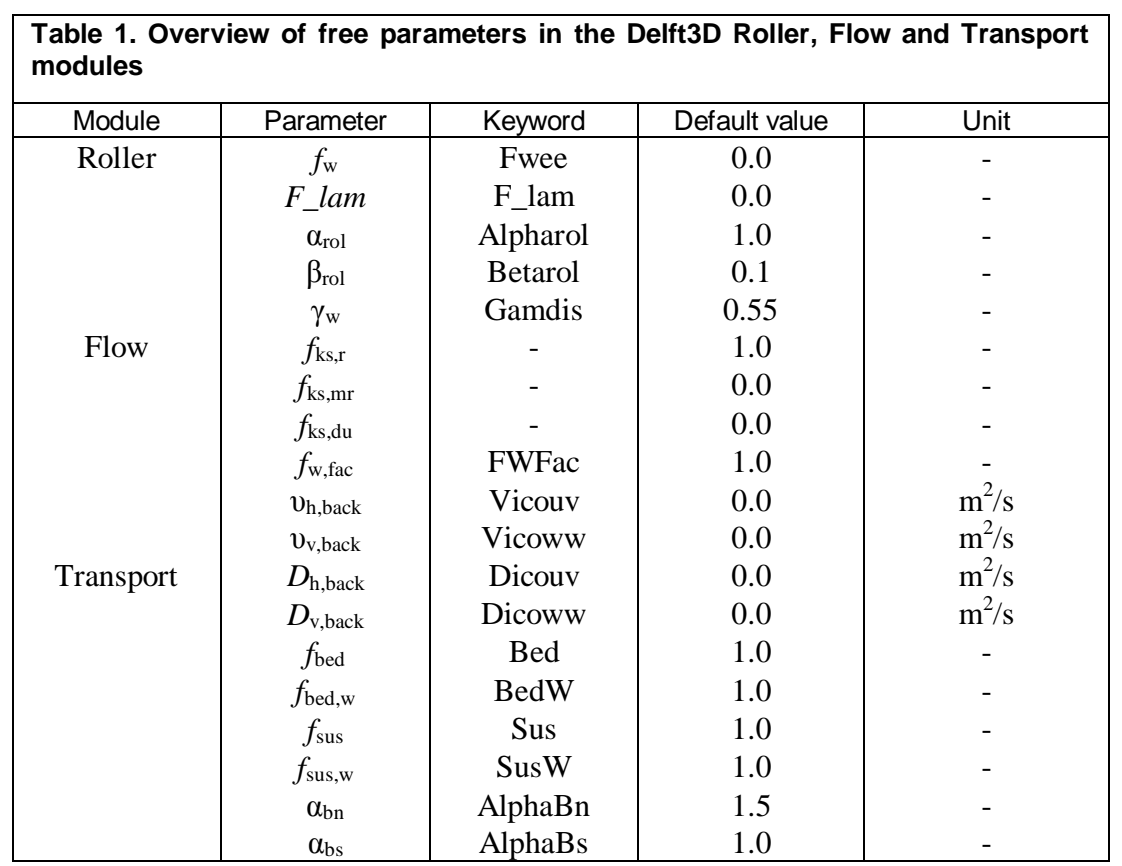

\section{CALIBRATION PROCEDURE}

\section{Purpose of the calibration tool}

Process or physically based models are simplified representations of natural systems, containing equations that express scientifically accepted principles, for instance, continuity and energy and/or momentum conservation for particular (partial) processes and their manifestation on specific scales of interest. However, model predictions are only as reliable as the model assumptions, inputs, and parameter estimates. The presence of parameters in process-based models is a direct consequence of the need to simplify reality or even represent unknown or sub-scale processes as a simple black box term. Essentially, parameters (or actually parameterizations) account for lacking information in the model due to complexity, or non-described spatial and/or temporal variability of the processes considered. In general, model parameters do not represent measurable attributes of the study area and, therefore, their values have to be determined through calibration using input/output relationships. In this study, the purpose of the calibration tool is to determine in an efficient and effective way the optimum parameter setting of the Delf3D-MOR model to compute morphological evolutions in coastal areas with complex bottom topography.

The calibration tool should be effective i.e. the calibration methodology or strategy should be such that the evaluation measure is sensitive to changes in the Delft3D-MOR parameter settings, both for strongly varying and more smooth error surfaces (i.e. strong or weak response of the Delft3D-MOR solution to parameter variation). In general, interdependence between parameters reduces the effectivity and should be minimized by fixing or limiting the range of one of the parameters. Moreover, efficiency implies that a calibration of the Delft3D-MOR model should be possible within reasonable time to allow different combinations of parameter sets to be evaluated. This criterion puts requirements on various aspects of the calibration tool:

1. The calibration method should use only a limited number of model runs;

2. The calibration should only consider a limited number of parameters to be determined;

3. A basic Delft3D-MOR computation should not take too much time.

\section{Measure of calibration quality}

Regarding morphodynamics, the primary variable solved by Delft3D-MOR is the bed level. Such an integral quantity allows for a quantitative evaluation. During the calibration process, a cost function (i.e. the criterion that must be minimized to find an estimate of uncertain model parameters) will be expressed in terms of the integral quantities $\xi$, i.e. the bed levels $z_{b}$, as available from measurements on the one hand, and from Delft3D-MOR model computations on the other hand. 
In a model calibration, the computed values are compared to observations. The latter are here denoted by $\hat{\xi}$. This comparison of model and observations is often based on the residuals $\hat{\xi}-\xi$. In practice a least squares criterion is used in the calibration process. For the associated cost function, $J(-)$, this will lead to the following generic formulation:

$$
J(\vec{\Theta})=\sum_{n=1}^{N} w_{n}\left(\xi_{n}(\vec{\Theta})-\hat{\xi}_{n}\right)^{2}
$$

While $\xi_{n}(\vec{\Theta})$ denotes the model prediction, the corresponding measured value is denoted by $\hat{\xi}_{n}$. The model predictions $\xi_{n}(\vec{\Theta})$ are a function of $P$ uncertain model parameters $\vec{\Theta}=\left(\Theta_{1}, \Theta_{2}, \ldots, \Theta_{P}\right)$ and these $\Theta_{1, \ldots, P}$ are the estimates of the model parameters. $N$ is the total number of measurements and is in this case the number of selected grid cells. The coefficient $w_{n}$ is a weight assigned to the $n$-th measurement. These weights can be different. They allow us to give more weight to the results obtained in the active bar zone v.s. the ones obtained in the swash zone (limit of validity of the shallow water equations).

\section{The DUD method}

Existing reviews (e.g., Sorooshian and Gupta, 1995; Lewis et al., 2000) show a bewildering number of calibration techniques. Despite the large amount of techniques, some sort of classification and a selection can be made.

The DUD (Doesn't Use Derivatives) technique of Ralston and Jennrich (1978) has been selected. The DUD is a directional minimization method. The main issue in the algorithm is that search directions are repeatedly computed on the basis of a linearization of the model response functions $\xi_{n}(\vec{\Theta})$ in Equation (1). This linearization is in the form of a so-called secant approximation of the $\xi_{n}(\vec{\Theta})$, rather than computing a tangent plane as commonly used in gradient descent techniques. The DUD method operates as follows:

1. The model must be evaluated for $P+1$ (mutually linearly independent) parameters $\vec{\Theta}^{(1)}, \vec{\Theta}^{(2)}$, $\ldots, \vec{\Theta}^{(P)}, \vec{\Theta}^{(P+1)}$. As an example, in case the method is applied to optimize two model parameters $\alpha$ and $\beta$, three simulations are then carried out, with parameters $\left[\alpha_{0}, \beta_{0}\right],\left[\alpha_{0}+d \alpha, \beta_{0}\right]$ and $\left[\alpha_{0}, \beta_{0}+d \beta\right]$, respectively.

2. A search direction is then computed, and a (one dimensional) line minimization procedure is carried out. The search direction results in the determination of a set of parameters $\left[\alpha_{1}, \beta_{1}\right]$ to be tested.

3. In fact, the iteration step may require a few more model evaluations to find a new and better estimate. The procedure is therefore repeated using the last $P$ sets, that provide the lowest cost functions over the last $P+1$ sets, until a minimum is found.

4. Still, when no minimum is found, a line search is performed along the segment that provides the lowest cost functions. In this way, the DUD method is a (i) local, (ii) directional, and (iii) iterative minimization method.

The number of iterations is model dependent, and will be about $P$. In this way a reasonable estimate for the total number of required model evaluations is expected to be about 5 to 8 times $P$. This number will not be limiting the practical feasibility of the DUD method, for a Delft3D-MOR application.

Considering functional and practical requirements, the DUD-minimisation algorithm of Ralston and Jennrich (1978) satisfies all the demands for Delft3D-MOR calibration. In fact,

1. The technique is optimally designed for least squares minimization criteria

2. Although gradient free (DUD stands for Doesn't Use Derivatives), the method algorithm, and more importantly its computational efficiency is comparable to those of quasi-Newton (or, in other words, variable metric) gradient descent techniques. 


\section{TWIN EXPERIMENT}

\section{Model set-up}

The hydrodynamics, sand transport rates and bed level changes are simulated with a twodimensional vertical (2DV) Delft3D model. Wave transformation follows from the Roller module and Snell's law, both part of Delft3D-FLOW.

The derived bathymetry and boundary conditions are representative for Egmond-aan-Zee, which is located in the northern part of the $120 \mathrm{~km}$ long Holland coast that runs from Hoek van Holland in the South to Den Helder in the North. The nearshore morphology can be considered as alongshore uniform and is characterized by two subtidal breaker bars and one intertidal swash bar.

An orthogonal flow grid is constructed, that that covers a $1500 \mathrm{~m}$ long cross-shore profile. This includes the upper shoreface, surf zone and beach with water depths up to $12 \mathrm{~m}$. The cross-shore grid resolution increases from $40 \mathrm{~m}$ at the seaward boundary to $20 \mathrm{~m}$ in the surfzone. The number of vertical computational layers equals 12 with a high resolution near the bed decreasing towards the water surface.

The tidal forcing is accounted for by a so-called morphological tide, which consists of a single semi-diurnal tidal cycle (with a duration of $12.5 \mathrm{hrs}$ ) representing the average long-term situation. Water levels are imposed at the offshore boundaries and water level gradients at the lateral boundaries. The wave climate is represented by four wave conditions ensuring identical yearly gross longshore transports by scaling the selected wave conditions (see Van Duin et al., 2004; Walstra et al., 2004).

Every flow time step (12 s) the waves, currents, sand transport rates and bed level changes are computed. To reduce computational time, the bed level changes are multiplied with a morphological scale factor (Morfac); such that, after one time step $\Delta \mathrm{t}$, modeled morphological changes over a period equal to Morfac* $\Delta \mathrm{t}$ are actually computed. This is valid since changes in morphology occur on a much longer time scale than changes in hydrodynamics (Roelvink, 2006).

The wave conditions are run serially. The first wave condition is applied to the initial bathymetry. The resulting bathymetry (after one morphological tide) feeds into the simulation with the next wave condition. This is repeated until four model simulations (one for each wave condition) have been carried out. In this way, one year of morphological change is simulated.

\section{Test of the DUD technique}

The calibration environment (DUD executable communicating with Delft3D-MOR model) has been tested in the so-called twin experiment. The objectives of the tests are:

1. To check that the calibration procedure works properly. This check includes the assessment of the functioning of the calibration method itself, and of the communication facility between the DUD environment and the Delft3D model,

2. To check, through the application, that the DUD method is (at least) one of the suitable methods that can be applied for a Delft3D model automated calibration,

3. To evaluate the computational demand,

4. To define, through the application, the number of model parameters that should be calibrated, and which ones,

5. To discuss the ability of the calibration environment to find the lowest Cost Function for model parameters identical to the targets

The test is performed with defining the observed profile as the computed one obtained for parameter settings chosen with their standard values (Walstra et al., 2004; Brière \& Walstra, 2006).

Thus, $\left[\beta_{\mathrm{rol}}=0.05 ; F_{-}\right.$lam $\left.=-2 ; \alpha_{\mathrm{bs}}=1 ; \alpha_{\mathrm{bn}}=1.5 ; f_{\mathrm{sus}}=1 ; f_{\mathrm{sus}, \mathrm{w}}=0.3 ; f_{\mathrm{ks}, \mathrm{r}}=f_{\mathrm{ks}, \mathrm{mr}}=1\right]$ are used as parameter settings. These parameters are selected because they were evaluated as influencing the morphological developments. Then these parameters are fixed at different but realistic values. Several tests have been carried out, using a different number (i.e. 1 to 5) of free parameters to be tested, different initial values for the free parameters, and different values for the initial parameter perturbations.

\section{Results}

For a sake of brevity, only the tests with two free parameters are discussed in this section. It has been generally observed that : 
1. In some cases, the two free parameters converge to the targeted values. This behavior is found in case independent free parameters are considered (e.g. $\beta_{\text {rol }}$ with $f_{\mathrm{ks}}$ ). Still such a behaviour occurs also when strongly interdependent parameters are considered (e.g. $f_{\text {sus }}$ with $f_{\text {sus,w }}$ ).

2. In other cases, the two free parameters do not converge to the targeted values. Two different cases can be distinguished: 1) the tests with varying $\alpha_{\mathrm{bs}}$ and $\alpha_{\mathrm{bn}}$ show that the morphological developments are quite insensitive to variations in these two parameters; 2) The test with $\beta_{\text {rol }}$ and F_lam varying, on the other hand, shows that the targeted values can not be reached although the final morphological development is different than the "observed" profile (Figure 1, left panel). A local minimum in the Cost function is found, and the variations in the free parameters from one simulation to another are around this local minimum. Consequently the absolute minimum can not be found. The interdependency of the two parameters explain the difficulty to converge to the targeted values. In case a restart procedure is set-up (Figure 1, right panel) with initial perturbations set to allow values around the targeted ones, the absolute minimum can finally be found.
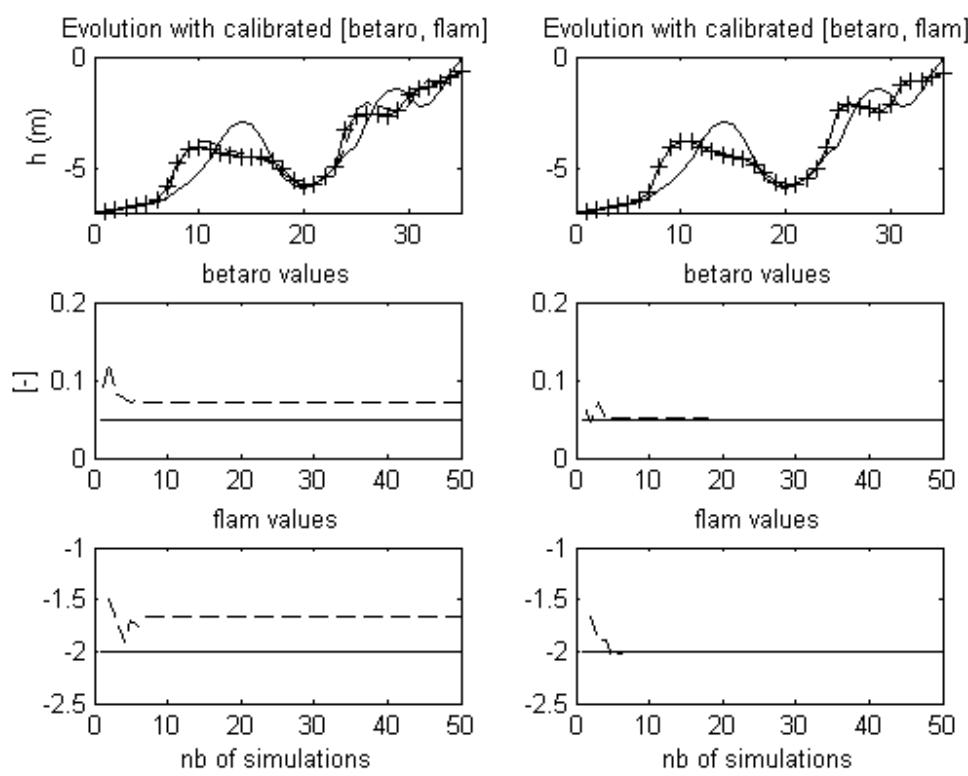

Figure 1. Final morphological developments for $\beta_{\mathrm{rol}}$ and $\boldsymbol{F}_{-}$lam varying parameters. Middle and low panels show the values over the calibration procedure, with the dashed and solid lines as the varying parameter and the targeted value, respectively.

\section{Discussion}

Respect to the functional/algorithmic issues underlying the selection of a feasible calibration technique, the aspects of implementation are not of primary importance since the Delft3D-MOR model has been treated as a mathematical function of the parameters. On request of the calibration technique, the numerical model produce its response to a variation of the model parameters. This response of the numerical Delft3D model is merely used by the calibration technique to adapt the Delft3D parameter settings and activate a new Delft3D model evaluation. As a result, the communication between the calibration technique and the numerical model is relatively simple, and the tests have proven that this communication performed well. Still, from a practical point of view, it means that the existing calibration environment for Delft3D can be easily extended to other (generic) calibration techniques, if necessary.

For the testing of the Delft3D model in the twin experiment, the computational time for a model evaluation is in the order of 20 minutes. Moreover, a sufficiently accurate estimate for the model parameters can be found in less than 50 simulations. The model computational time is then not a limiting factor. However, this suggests that for other practical applications (e.g. Egmond-aan-Zee Field case), the computational time should not be increased significantly.

For the twin experiment, the number of model parameters in the Delft3D calibration varied from 1 to 5 , that can be assumed to be relatively small (especially when compared to calibration or assimilation of large scale dynamic hydraulic and meteorological numerical models which may 
involve hundreds or even thousands of uncertain model parameters). This number is sufficiently small to be not really discriminating for more practical applications (e.g. Egmond-aan-Zee Field case).

The twin experiment has shown that, for Delft3D-Mor, the probability that a local minimum is identified rather than a global minimum is quite significant. Such a result can be seen as a failure of the calibration test. However, the tests have proven that the communication between the DUD environment and the Delft3D model performed well, and more important that the DUD method operates as requested. It is therefore not compelled to reconsider the application of the DUD method for the calibration purpose, but better necessary to improve the methodological process.

In this configuration, the tests have shown that the most satisfying set-up for a Delft3D model calibration consists of the optimization of a limited number of independent model parameters. Moreover, it has been shown that these model parameters should be chosen such that the Delft3D model results are sensitive to changes in the parameter settings. It is therefore recommended to perform the calibration of Delft3D-Mor by means of optimizing the settings of parameters $\beta_{\text {rol }}$ and $f_{\text {sus,w. }}$ Typical initial values can be the standard ones.

\section{EGMOND-AAN-ZEE FIELD CASE}

\section{Model set-up}

The parameter optimization tool is applied to the case study of Egmond-aan-Zee (The Netherlands). This site has been widely investigated in the past and a large amount of field information is available (e.g COAST3D - EGMOND, 2002).

Egmond-aan-Zee is located in the central part of the Dutch coast. The mean tidal range varies between $1.2 \mathrm{~m}$ in the neap cycle to 2.1 in the spring cycle. The tidal peak current is about $0.5 \mathrm{~m} . \mathrm{s}^{-1}$ in the offshore zone, with the flood current to the North slightly larger than the ebb current directed to the South. The mean monthly offshore wave height has a seasonal character, and varies from about 1 $\mathrm{m}$ in the summer months (May to August) to about 1.5 to $1.7 \mathrm{~m}$ in the autumn and winter (October to January). The mean wave height can reach $5 \mathrm{~m}$ at $15 \mathrm{~m}$ depth, during major storms.

The beach width is about $100 \mathrm{~m}$ to $125 \mathrm{~m}$ with a slope between 1:30 and 1:50. Two main longshore breaker bars run parallel to the shoreline most of the time. The inner bar is located $200 \mathrm{~m}$ from the shoreline at $2 \mathrm{~m}$ below the mean sea water level (MSWL), whilst the crest of the outer bar is located at about $500 \mathrm{~m}$ from the shore at $4 \mathrm{~m}$ below the MSWL. The inner bar is separated from the outer one by a wide trough. The area is characterized by medium well-sorted sands $(0.25$ to $0.5 \mathrm{~mm})$, although in the trough between the inner and outer bars, the sand is coarser (>0.5 $\mathrm{mm}$ ) and has a moderate sorting. The cross-shore slope amounts to 1:100, and the median grain size is about $0.2 \mathrm{~mm}$.

At Egmond-aan-Zee, a combination of bathymetry data, wave data, and tidal levels were collected during the period 16 October $1998-7$ November 1998. Bathymetry surveys were performed on the 16th of November, 19th of November, 4th of November, and 7th of November, approximately between $-6 \mathrm{~m}$ water depth and the beach. Bathymetry data were extrapolated towards the sea with older measurements, in order to move the boundary of the model further from the area of interest. Wave data (root mean square wave height, peak period, and direction) and water levels were available every hour. The period was characterized by severe wave conditions with two storms with a wave height of about 4 meters. This induced an offshore bar migration of about $40 \mathrm{~m}$ of the outer bar and of about 25 $\mathrm{m}$ of the inner bar.

The Delft3D model is implemented on a grid with resolution ranging between $100 \mathrm{~m}$ at the sea boundary and approximately $10 \mathrm{~m}$ at the coastline. One application with two varying calibration parameters ( $\beta_{\text {rol }}$ and $\left.f_{\text {sus, } w}\right)$ is carried out. These parameters are selected in order to have a direct handle both on the hydrodynamics, through the calibration parameter $\beta_{\text {rol }}$, and on the sediment transport through the parameter $f_{\text {sus,w }}$. Among the parameters directly responsible for the sediment transport, the parameter $f_{\text {sus,w }}$ is chosen as calibration parameter due to the fact that this parameter is directly linked to a less known physical phenomenon.

The cost function used to evaluate the difference between model prediction and observation in the bathymetry evolution is computed considering only grid points between $-6 \mathrm{~m}$ and $-2 \mathrm{~m}$ water depth. This choice is undertaken in order to consider in the cost function only grid points defining the position of the bars. The offshore side of the outer bar (below - $6 \mathrm{~m}$ ) is not considered in the cost function due to the fact that the exact bathymetry measurements for this specific zone are not available from all the observations. The simulation is carried out assuming as a target the bathymetries measured on the 19th of November, 4th of November and 7th of November. In other words, the DUD 
method tends to minimize the cost function which takes into account the difference between model and measured bathymetry at these three different days.

The hydrodynamics, sand transport rates and bed level changes are simulated with a 2DV Delft3D model. It should be mentioned that the expression of Ruessink et al. (2003) is used for the wave breaking index $\left(\gamma_{\mathrm{w}}\right)$. The breaker delay is switched off.

\section{Results}

Results from the calibration are shown in Figure 2. The figure shows the initial bathymetry (continuous black line), two final simulated bathymetries respectively obtained using standard and calibrated parameter settings (circles and plus) and the final measured bathymetry (dashed black line). The final simulated bathymetry shows an exaggerated flattening of the bars (especially of the outer bar) when considering the result obtained by using the standard values for the two free parameters (plus in figure 2). Moreover, the offshore migration of the bars is over-predicted. The excessive flattening of the bars has been already observed in numerical simulations carried out by Walstra et al. (2008).

When calibrating the free parameters, the $\beta_{\text {rol }}$ parameter decreases from 0.05 and converges to the value of 0.015 , while the parameter $f_{\text {sus,w }}$ moves as well away from its initial value $(0.3)$ to converge to a value of about 2.5. The best representation of the bar dynamics with Delft3D is obtained by decreasing the value for $\beta_{\mathrm{rol}}$, that results mainly in more pronounced inner and outer bars, and by simultaneously increasing the onshore directed transport component, in this case by increasing the value of $f_{\text {sus,w }}$, that scales the wave-related suspended transport. Still, it should be mentioned that both the $\beta_{\text {rol }}$ and the $f_{\text {sus,w }}$ parameters are, respectively, far lower and higher than the ones generally adopted when using Delft3D.

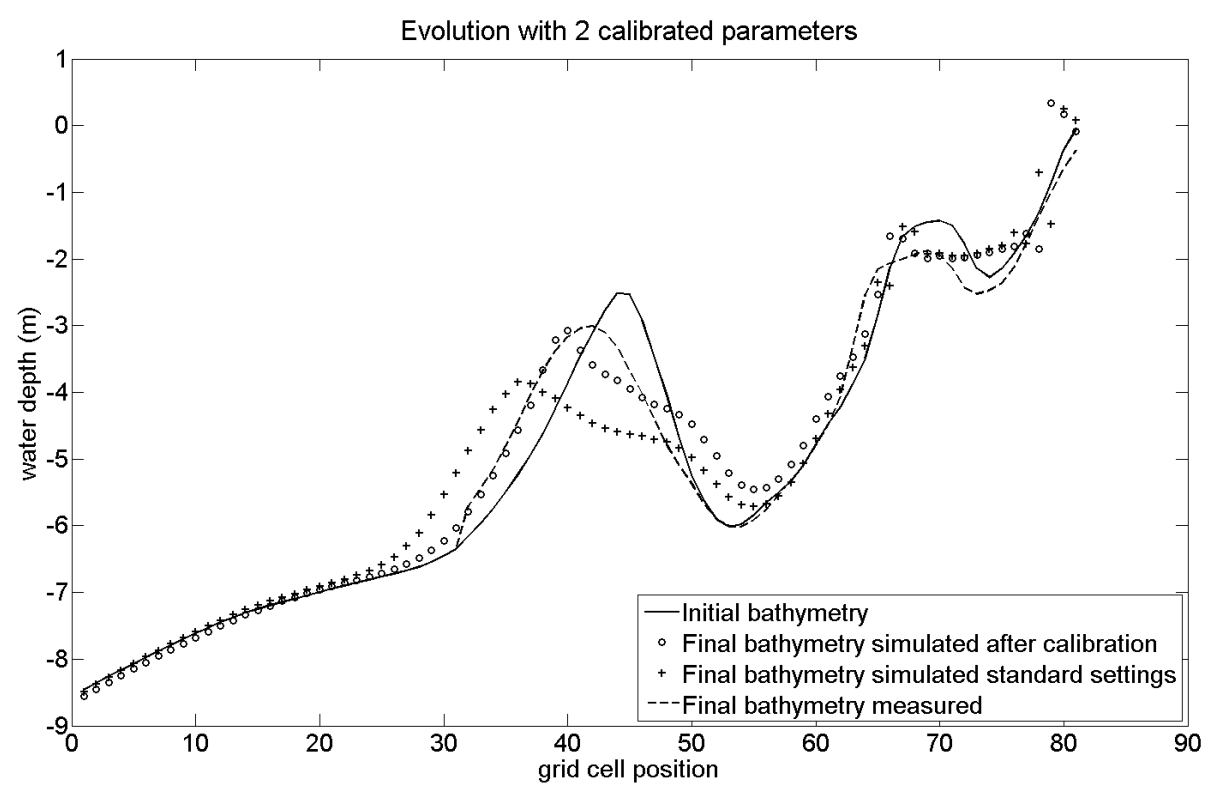

Figure 2. Cross-shore profile at Egmond-aan-Zee

\section{Discussion}

The DUD method is applied to find the free model parameter settings that give the best match between modeled and measured bathymetries along a cross-shore transect at Egmond-aan-Zee. A simulation adopting $\beta_{\text {rol }}$ and $f_{\text {sus,w }}$ as calibration parameters has been carried out. The calibration shows that more accurate results, with respect to the ones obtained with standard parameter settings, could be obtained by enhancing the wave-related transport through very high value of the parameter $f_{\text {sus,w. }}$. This could be due to a number of reasons. Is Delft3D underestimating the onshore-directed transport or is it trying to compensate overpredicted values of the offshore related transport? Several scientific questions arise which will need further investigation. In particular: 
1. Does the optimum parameter setting only mimic accurately the bar evolution or is it also reproducing the forcing responsible to these morphodynamic changes (in first place wave breaking, cross-shore currents, bedload and suspended load)?

2. Moreover, the calibration resulted in extremely low values of $\beta_{\text {rol }}$ and high values for $f_{\text {sus, } w}$ It will therefore be opportune in further work to constrain some of the used parameters into ranges which are physically acceptable.

\section{CONCLUSIONS}

The Roller, Flow and Transport modules of Delft3D contain 19 main free parameters. Review of previous studies on the modeling of the morphological evolution of breaker bar dynamics and shoreface nourishments showed that 13 of these were actually used for model calibration. There is agreement across different studies on the settings of 9 of these parameters. There is no general agreement on values for the roller slope $\left(\beta_{\text {rol }}\right)$, wave height-to-depth ratio $\left(\gamma_{w}\right)$, scaling of the waverelated suspended load $\left(f_{\text {sus,w }}\right)$ and the coefficients for the bed slope effects $\left(\alpha_{\mathrm{bs}}\right.$ and $\left.\alpha_{\mathrm{bn}}\right)$.

Within this study, the communication between a suitable calibration technique and a 2DV Delft3D model was developed. Among all the calibration techniques considered, the DUD-method of Ralston and Jennrich (1978) is found to fit best our purpose. The number of required model evaluations is about 5 to 8 times the number of uncertain model parameters. The DUD-method is optimally designed for least squares criteria. The selected cost function (i.e. the criterion that must be minimized to find an estimate of uncertain model parameters) is expressed in terms of integral quantities, i.e. the bed levels, as available from measurements on the one hand, and from Delft3D model computations on the other hand.

The calibration environment (DUD executable communicating with Delft3D-MOR model) is tested based on a so-called twin experiment. This experiment shows that the probability to identify a local minimum rather than a global minimum is quite significant. However, the experiment also shows that the calibration procedure operates as requested. In this configuration, the tests show that the most satisfying set-up for a Delft3D model calibration is the optimization of a limited number of independent model parameters. In particular, it is shown that these model parameters should be chosen such that the Delft3D model results are sensitive to changes in the parameter settings. It is therefore recommended to perform the calibration of Delft3D-MOR by means of optimizing the settings of parameters $\beta_{\mathrm{rol}}, f_{\text {sus,w }}$ (and potentially $f_{\mathrm{ks}, \mathrm{r}}$ ). Typical initial values can be the standard ones.

The DUD method in combination with a 2DV Delft3D model is validated using one month of bathymetric data collected along a cross-shore transect at Egmond-aan-Zee. Bathymetry data collected at three different dates were used as targets to compute the best parameter settings. The procedure was set-up using $\beta_{\text {rol }}$ and $f_{\text {sus,w }}$ as calibration parameters. The values obtained for $\beta_{\text {rol }}$ and $f_{\text {sus,w }}$ are respectively equal to 0.015 and 2.5 .

The calibration procedure shows that more accurate results in terms of outer bar dynamics (i.e. bar position, bar height ...), are obtained through the calibration of two independent parameters instead of using the Delft 3D standard parameter settings.

However, the value for $\beta_{\text {rol }}$ is rather low when compared to what is usually adopted in literature, showing the necessity of further research in the description of the wave breaking processes. On the other hand, the value of 2.5 for $f_{\text {sus, } w}$ is far higher than what is usually taken, showing the necessity in the model to increase the onshore-directed transport to have a good representation of the morphological changes.

\section{RECOMMENDATIONS}

For the application of the DUD calibration technique, initial guesses must be provided for the uncertain model parameters. These initial guesses should be based on physical knowledge and modeling experience. However, the experiences in this study suggest that the calibration result is highly dependant on these initial guesses. It is therefore crucial to select appropriate initial guesses for the uncertain model parameters before starting the calibration procedure. The suitability of the initial guesses should be verified by means of a few preliminary model evaluations. To facilitate the selection of appropriate initial guesses, it is recommended to develop an automated tool for a manual prescanning of the model parameter space. The idea is that a user will specify an arbitrary set of combinations of the parameters, and in a batch procedure the model is then evaluated for the combination of the model parameters. By comparing the results, the sensitivity of the model can be verified, and a reasonable estimate may become available for the range where the optimal parameter 
settings must be expected. This tool should be seen as an auxiliary, automated, and efficient preprocessing facility.

In this paper, the calibration tool is validated using only one set of field data. Further work is necessary using additional datasets to quantify the variability of the calibration parameters in function of the location and the wave conditions. Those findings could be then used to provide some simple "calibration recipes", which the user could apply to set up a fast Delft3D run, even when no field data are available.

Finally, the investigation focused in finding the optimum set of parameters producing the best agreement between modeled results and measurements in terms of bed level changes. However, it is advisable to check whether these parameters will also reproduce realistically other physical variables (wave height, cross-shore and longshore currents, bedload and suspended load).

\section{ACKNOWLEDGMENTS}

This study has been performed in the framework of the Kustlijnzorg program, and funded by the Dutch Ministry of Transport and Public works.

\section{REFERENCES}

Bagnold, R. A., 1966. An approach to the sediment transport problem from general physics. U.S. Geological Survey Professional Paper 422-I.

Battjes, J.A., and J.P.F.M. Janssen. 1978. Energy loss and set-up due to breaking of random waves, Proceedings of $14^{\text {th }}$ International Conference on Coastal Engineering, ASCE, 466-480.

Brière, C., Walstra., D. J. R., 2006. Modelling of bar dynamics. Report Z4099, WLIDelft Hydraulics, The Netherlands.

COAST3D-EGMOND, 2002. The behaviour of a straight sandy coast on the time scale of storms and season; process knowledge and guidelines for coastal management. Edited by L.C. van Rijn, B.G. Ruessink, and J.P.M. Mulder. 580 pp.

Giardino, A., Brière, C. and van der Werf, J.J., 2010. Morphological modelling of bar dynamics with Delft3D, the quest for optimal free parameter settings. Report 1200731, Deltares, The Netherlands.

Lesser,G.R., Roelvink, J.A., van Kester, J.A.T.M., Stelling, G.S., 2004. Development and validation of a three dimensional morphological model. Journal of Coastal Engineering, 51, 883-915.

Lewis, R.M., V. Torczon and M.W. Trosset, 2000. Direct search methods: then and now. Journal of Computational and Applied Mathematics, 124, 191-207.

Ralston, M.L. and R.I. Jennrich, 1978. Dud, a derivative-free algorithm for nonlinear least squares. Technometrics, 20, 7-14.

Reniers, A.J.H.M., Roelvink, J.A., Thornton, E.B., 2004. Morphodynamic modeling of an embayed beach under wave group forcing. Journal of Geophysical Research, 109.

Roelvink, J.A., 1993. Dissipation in random wave groups incident on a beach, Coastal Engineering, 19, Issues 1-2, 127-150.

Roelvink, J.A., Meijer, Th.J.G.P., Houwman, K., Bakker, R., Spanhoff, R., 1995. Field validation and application of a coastal profile model. Proceedings Coastal Dynamics Conference, Gdansk, Poland, pp. 818-828.

Roelvink, J. A., 2006. Coastal morphodynamic evolution techniques. Coastal Engineering 53: 277287.

Ruessink, B.G., Kuriyama, Y., Reniers, A.J.H.M., Roelvink, J.A., Walstra D.J.R., 2007. Modeling cross-shore sandbar behaviour on the timescale of weeks. Journal of Geophysical Research, 112, $1-15$.

Sorooshian, S. and V.K. Gupta, 1995. Model calibration. In: Computer models of watershed hydrology. Ed. V. Singh, pp. 23-68.

Van Duin, M.J.P., Wiersma, N.R., Walstra, D.J.R., Van Rijn, L.C., Stive, M.J.F., 2004. Nourishing the shoreface: observations and hindcasting of the Egmond case, The Netherlands. Journal of Coastal Engineering, 51: 813-837.

Van Rijn, L. C., 1993. Principles of sediment transport in rivers, estuaries and coastal seas. Aqua Publications, The Netherlands. 
Van Rijn L.C., 2007a. Unified view of sediment transport by currents and waves. I: Initiation of motion, bed roughness, and bed-load transport. Journal of Hydraulic Engineering 133 (6): 649667.

Van Rijn L.C., 2007b. Unified view of sediment transport by currents and waves. II: Suspended transport. Journal of Hydraulic Engineering 133 (6): 668-689.

Walstra, D.J.R., Van Ormondt, M., Roelvink, J.A., 2004. Shoreface Nourishment Scenarios, WL | Delft Hydraulics report Z3748.21.

Walstra, D.J.R., Brière, C.B., Cohen, A.B., Van Dongeren, A.P., Elshoff, I.J.P., Hoyng, C. Van Ormondt, M., Quartel S., De Sonneville, B., Tonnon, P.K., Uunk, L., 2008. Monitoring and modelling of a shoreface nourishment. Report Z4479, Deltares, The Netherlands. 